Kansas State University Libraries

New Prairie Press

\title{
ANALYSIS OF REPEATED MEASURES DATA
}

Ramon C. Littell

Follow this and additional works at: https://newprairiepress.org/agstatconference

Part of the Agriculture Commons, and the Applied Statistics Commons

\section{(c) $($ ) $\ominus$}

This work is licensed under a Creative Commons Attribution-Noncommercial-No Derivative Works 4.0 License.

\section{Recommended Citation}

Littell, Ramon C. (1990). "ANALYSIS OF REPEATED MEASURES DATA," Conference on Applied Statistics in Agriculture. https://doi.org/10.4148/2475-7772.1427

This is brought to you for free and open access by the Conferences at New Prairie Press. It has been accepted for inclusion in Conference on Applied Statistics in Agriculture by an authorized administrator of New Prairie Press. For more information, please contact cads@k-state.edu. 


\title{
ANALYSIS OF REPEATED MEASURES DATA
}

\author{
RAMON C. LITTELL \\ Department of Statistics \\ Institute of Food and Agricultural Sciences \\ University of Florida \\ Gainesville, FL 32611
}

\begin{abstract}
Data with repeated measures occur frequently in agricultural research. This paper is a brief overview of statistical methods for repeated measures data. Statistical analysis of repeated measures data requires special attention due to the correlation structure, which may render standard analysis of variance techniques invalid. For balanced data, multivariate analysis of variance methods can be empioyed and adjustments can be applied to univariate methods, as means of accounting for the correlation structure. But these analysis of variance methods do not apply readily with unbalanced data, and they overlook the regression on time. Regression curves for treatment groups can be obtained by fitting a curve to each experimental unit, and then averaging the coefficients over the units. Treatment groups can be compared by applying univariate and multivariate methods to the group means of the coefficients. This approach does not require knowledge of the correlation structure of the repeated measures, and an approximate version of it can be applied with unbalanced data.
\end{abstract}

Key words: repeated measures, analysis of variance, regression, random coefficient model

\section{INTRODUCTION}

The expression "repeated measures data" refers to multiple measurements on the same unit. In the general sense, the term "unit" could refer to an experimental unit in a designed experiment, a sampling unit in a sample survey, or a subject in a retrospective study. In most situations, the repeated measures are over time, but they could be over space as well. The term "point" will be used to refer to a point in time or a point in space.

Data with repeated measures occur in virtually all fields of agricultural research. Perhaps the most frequent occurrence is in growth measurements of plants or animals over time. Other examples of repeated measures over time include crop yields from multiple harvests on experimental plots, daily milk yields from individual cows, and weekly livestock prices at individual auction markets. Examples of repeated measures in space are moisture determinations at several depths in soil core samples, measurements of a pollutant at numerous points on a line transect, and amounts of spray deposition at several sites within citrus trees.

For the sake of convention and uniformity, we shall use terminology for repeated measures in time. Also, we shall use the term "unit" to refer to the sampling unit or experimental unit, whichever the case may be.

Studies with repeated measures data have traditionally been analyzed as "split plot in time" experiments. This means that a split plot analysis of variance is performed with the "units" on which the repeated measures are taken treated as main-plot units and the "units" at particular points in time treated as a sub-plot units. This approach has many shortcomings. The repeated measures must be taken at the same time points on each unit, and there must be a certain correlation structure between the repeated measures on the same unit in order for analysis of variance $F$ statistics to be valid. Also, trends over time are commonly ignored when the "split plot in time" approach is used. In recent years, many alternative statistical methods have been developed.

This paper provides a brief survey of several methods for analysis of repeated measures data. It draws from recent work of several authors and the facilities of certain computer program 
packages. Section 2 discusses analysis of variance of "balanced" repeated measures, in which all units have complete data at a specified set of points. Methods for this case are well developed and several program packages provide adequate computing facility for these methods. Disagreement exists, however, on the merits of various methods, and when one is preferred to another. Section 3 discuses a regression approach to repeated measures data in the balanced case, treating time as a regression variable. Section 4 discusses analysis of "unbalanced" repeated measures data, in which case different units have data at different points. Methods of analysis for this situation are much more complicated, and generally require some degree of approximation.

\section{ANALYSIS OF VARIANCE FOR BALANCED REPEATED MEASURES DATA}

Repeated measures data will be called "balanced" if every unit has complete data at the same time points. For balanced repeated measures data, the traditional method is the split-plot in time analysis of variance. This is also called the "univariate" repeated measures analysis of variance.

An example of balanced repeated measures data is given in Freund, Littell and Spector (1986). These data came from a large study of the eff:cts of nutrition and exercise on physical strengths of geriatric citizens. Concern here is with the exercise aspects of the study, which involved three weight training programs to which subjects were randomly assigned. Each subject's strength, as determined by the amount of weight the subject could lift, was measured every other day for two weeks. The first program was a control, in which no training was employed (CONT). The second program utilized a weight training system in which the number of repetitions of the exercise was increased incrementally with time (RI). In the third program, the amount of weight was increased incrementally over time (WI). Data are shown in Table 1, with means and standard deviations in Table 2. Mean profiles for the three programs are plotted in Figure 1.

The exercise therapy study is typical of many repeated measures designs in which subjects are randomly assigned to "treatment" groups, and a response variable is measured repeatedly over time.

A model for data from this type of study is

$$
\mathrm{y}_{\mathrm{ijk}}=\mu_{\mathrm{ik}}+\mathrm{e}_{\mathrm{ijk}},
$$

where $y_{\mathrm{ijk}}$ is the response of the $\mathrm{jth}$ subject in the ith treatment at the kth time, and $\mu_{\mathrm{ik}}$ is the population mean for treatment $\mathrm{i}$ at time $\mathrm{k}$. The errors $\mathrm{e}_{\mathrm{ijk}}$ are assumed normally distributed with mean zero and $\mathrm{V}\left(\mathrm{e}_{\mathrm{ij}}\right)=\mathrm{V}$, where $\mathrm{e}_{\mathrm{ij}}=\left(\mathrm{e}_{\mathrm{ij} 1}, \ldots, \mathrm{e}_{\mathrm{ijt}}\right)$.

A key issue with repeated measures data is the structure of the covariance matrix V. Here are five particular structures in terms of mathematical conditions on $\sigma_{\mathrm{kk}}$, the element in row $\mathrm{k}$, column $\mathrm{k}$, of $\mathrm{V}$, that play a role in repeated measures data:

Structure

1. Unstructured

2. Spherical

3. Compound symmetric

4. Huynh-Feldt

5. Autoregressive no restrictions

equal variances

zero covariances

equal variances

equal covariances

unrestricted variances restricted covariance

covariance function of time interval between repeated measures
Mathematical Condition

$\mathrm{V}=\left(\sigma_{\mathbf{k k}^{\prime}}\right)$ positive definite

$\sigma_{\mathrm{kk}}=\sigma^{2}$,

$\sigma_{\mathrm{kk}}=0$

$\sigma_{\mathrm{kk}}=\sigma^{2}$,

$\sigma_{k \mathbf{k}^{\prime}}=\delta \sigma^{2}$

$\sigma_{\mathrm{kk}}=2 \tau_{\mathrm{k}}+\phi$

$\sigma_{\mathbf{k n}^{\prime}}=\tau_{\mathbf{k}}+\tau_{\mathbf{k}^{\prime}}$

$\sigma_{\mathrm{kk}^{\prime}}=\Theta_{\left|\mathrm{k}-\mathrm{k}^{\prime}\right|}$ 
A "univariate" analysis of variance (split plot in time analysis of variance) for the exercise therapy data is shown in Table 3. The asterisk next to the P-values for Time and Time*Program $F$ values is a flag that the validity of these $F$ tests depends upon $V$ having a certain mathematical structure. Many sources state that the required condition is compound symmetry, but in fact it is the Huynh-Feldt (H-F) condition. See Huynh and Feldt (1970). However, there is probably little practical distinction between the two conditions. The H-F condition is equivalent to any set of orthonormal contrasts of the repeated measures having a spherical distribution.

Both the compound symmetry and $\mathrm{H}-\mathrm{F}$ conditions are questionable from a practical point of view. There is little evidence to indicate that either of these conditions should occur in nature. A more realistic condition for repeated measures in time is the first order autoregressive structure. It accommodates the feature that measures close in time will be more highly correlated than measures far apart in time; in particular, covariance between two repeated measures is a function only of their distance apart in time.

Multivariate methods can be used to test the Time and Time*Treatment effects which do not require any particular structure of $\mathrm{V}$. These can be constructed by computing sets of contrasts among the repeated measures and applying multivariate analysis of variance techniques (Cole and Grizzle, 1966). Many of the multivariate tests can also be obtained directly by some computer programs; in particular, the REPEATED statement in the ANOVA and GLM procedures in the SAS System. The generality of the multivariate methods tends to come at a cost in statistical power. For the exercise therapy study, multivariate tests (based upon Pillai's trace) produce the results in Table 4.

It is not uncommon to obtain very different results regarding Time*Treatment interaction from the multivariate test and the univariate test. The univariate test in Table 3 produced $\mathrm{P}=0.0005$ and the multivariate test in Table 4 produced $\mathrm{P}=0.1943$. One must know which, if either, of these P-values to believe. A general approach for analysis of variance of repeated measures data is to use the univariate tests if the H-F condition holds, and use the multivariate tests if the H-F condition does not hold. The problem is in knowing whether the $\mathrm{H}-\mathrm{F}$ condition holds.

Tests of significance have been recommended to determine if the H-F condition holds; i.e. to determine if the mathematical conditions are met which are required for validity of the univariate analysis of variance $F$ statistics for the Time and Time*Treatment interaction. One of these tests, which is available in the SAS System under the name "Mauchly's criterion," is a likelihood ratio test for the null hypothesis that the H-F condition holds versus the alternative hypothesis that $\mathrm{V}$ is unstructured. Tests for the validity of the $\mathrm{H}-\mathrm{F}$ condition have recently been discredited, however. (See Looney and Stanley, 1989). This leaves this general approach of choosing between the univariate and multivariate tests in a state of limbo.

Alternatively, adjusted versions of the univariate tests can be employed. These procedures entail adjusting the significance probability of the univariate analysis of variance $F$ tests according to the degree of departure from the H-F conditions. Box (1954) proposed a measure $\epsilon$ of the degree of departure from the H-F conditions. The value of $\epsilon$ is between $(t-1)^{-1}$ and 1 , where $t$ is the number of time points. The value $\epsilon=1$ indicates the $H-F$ condition holds, while decreasing values of $\epsilon$ indicate increasing departure from the H-F condition. Box (1954) showed that significance probabilities of the univariate $F$ tests for the Time and Time* Treatment effects can be adjusted by discounting the numerator and denominator degrees of freedom by multiplying them by $\epsilon$. Thus $\epsilon=1$ would produce no adjustment of the P-value. On the other extreme, $\epsilon=(t-1)^{-1}$ would produce the most severe adjustment, reducing the degrees of freedom for time from $t-1$ to 1 , for Time*Treatment from $(t-1)^{*}(a-1)$ to $a-1$ (where $a$ is the number of treatment groups), and for Error from $(n-a)^{*}(t-1)$ to $n-a$ (where $n$ is the number of units altogether). However, in practice one would not usually know the value of $\epsilon$. A conservative adjustment is to adjust the significance probabilities as if $\epsilon=(t-1)^{-1}$. Another approach is to estimate $\epsilon$ from the data. Greenhouse and Geisser (1959) and Huynh and Feldt (1970) proposed estimates of $\epsilon$. See Milliken and Johnson (1984) or Milliken (1990) for good discussions of these methods. Looney and Stanley (1989) make recommendations concerning a combination of the adjusted, unadjusted and multivariate tests for the Time and Time* Treatment effects. 
Univariate, multivariate and adjusted univariate P-values for the exercise therapy data are summarized in Table 5. All three tests for the Time effect are significant with $\mathrm{P}<0.0001$. However, this is the "main effect" of Time; that is, the effect of Time averaged across programs. The plot in Figure 2 suggests that the Time trends are not parallel. This is to be expected because RI and WI are active programs and should produce strength measurements which increase with Time, whereas CONT is inactive and should not result in strength increasing with Time. Therefore, the significance of the Time trend averaged across Programs is of little practical value. The nature of the Time*Program interaction is a more important issue. Recall that the univariate test for Time*Program has a P-value of 0.0005 and the multivariate test for Time* Program has a P-value of 0.1943 , giving conflicting conclusions from the two tests. Mauchly's test for the validity of the $H-F$ condition yielded $P<0.0001$, which indicates the $H-F$ condition does not hold. Therefore, the univariate P-value for Time*Program is unreliable. On the other hand, the multivariate test lacks the power to detect the Time* Program interaction that seems apparent in Figure 1. The Greenhouse-Geisser (1959) estimate of the discounting factor $\epsilon$ is 0.4233 . When the degrees of freedom of the univariate test are multiplied by 0.4233 , the resulting adjusted $\mathrm{P}$ value is 0.0130 . This $P$-value seems in agreement with the visual impression of $f$ interaction in Figure 2.

\section{REGRESSION METHODS FOR BALANCED REPEATED MEASURES DATA}

All of the analysis of variance methods discussed in the preceding section regarding tests of significance for the Time and Time* Treatment effects ignore a basic and essential feature of the variable Time; namely, that it is quantitative. Therefore, as in the case of any quantitative variable, a more effective method of analysis is usually obtained by employing some form of regression methodology. That is, one should think in terms of fitting curves to the Time variable. Typically, one would potentially have a different regression curve for each treatment (in this case, each Program).

The regression approach has many advantages. First, and perhaps most importantly, it directs the data analysis toward a major objective of the study by focusing on trends over time. Second, it provides a reduction in the number of parameters to be estimated; instead of the $t$ means $\mu_{\mathrm{i} 1}, \ldots, \mu_{\mathrm{it}}$ for the ith treatment, one would have only the parameters of the mean regression curve, typically two or three in number. Third, inference can be made in terms of the regression parameters. Hopefully, regression functions could be specified so that the parameters have some practical interpretation. Fourth, regression curves "smooth" the response over time. Estimates of differences between treatments can be calculated from fitted curves that will avoid the erratic conclusions often obtained from comparing treatment means at each time point. It is not uncommon, when estimating differences between treatment means at individual time points, to find the differences vacillate between "significant" and "not significant" from one time to the next due to the random variation in the means.

Regression curves can be fitted to individual units, providing estimates of the regression parameters for each unit. Then multivariate and univariate statistical analysis of the unit regression parameter estimates can be performed to determine in what manner characteristics of the regression curves are affected by the treatment groups. In terms of a model, we have

$$
\mathbf{y}_{\mathrm{ij}}=\mathbf{X} \beta_{\mathrm{i}}+\mathbf{e}_{\mathrm{ij}}
$$

where $\mathbf{y}_{\mathrm{ij}}$ is the vector of repeated measures for the $\mathrm{jth}$ unit in the ith treatment group, $\mathbf{X}$ is the matrix relating the repeated measures to the time points, $\beta_{\mathrm{i}}$ is the parameter vector for the ith treatment, and $\mathbf{e}_{\mathrm{ij}}$ is the error vector for the $\mathrm{jth}$ unit in the ith group. Fitting the model to each unit via ordinary least squares (OLS) yields

$$
b_{i j}=\left(X^{\prime} \mathbf{X}\right)^{-1} X^{\prime} y_{i j}
$$
as the vector of parameter estimates for unit $\mathrm{j}$ in group $\mathrm{i}$. Then $b_{\mathrm{ij}}$ has expectation $E\left(\mathbf{b}_{\mathrm{ij}}\right)=\beta_{\mathrm{i}}$, and
NewPrairiePress 
variance $\mathrm{V}\left(\mathbf{b}_{\mathrm{ij}}\right)=(\mathbf{X} \mathbf{X})^{-1} \mathbf{X}^{\prime} \mathbf{V X}\left(\mathbf{X}^{\prime} \mathbf{X}\right)^{-1}$. Therefore, the $\mathbf{b}_{\mathrm{ij}}$ estimates follow a multivariate normal distribution, which we write

$$
\mathbf{b}_{\mathrm{ij}} \sim \operatorname{MVN}\left(\beta_{\mathrm{i}},(\mathbf{X} \mathbf{X})^{-1} \mathbf{X} \mathbf{V X}(\mathbf{X} \mathbf{X})^{-1}\right)
$$

It follows that standard methods for the general multivariate linear model can be applied. (See Grizzle and Allen, 1969). This provides the basis for a seemingly ad hoc but effective methodology for inference. Standard multivariate analysis computer programs can be used to perform the analyses, taking the vector $b_{i j}$ of parameter estimates for the $j$ th unit in the ith group as the "observation" vector. In fact, as will be pointed out in the next section, there is some basis for using this approach even in the unbalanced case. A tremendous advantage of this ad hoc approach is that it does not require knowledge of $\mathrm{V}$. Therefore, questions of the specific form for $\mathrm{V}$ (compound symmetry, $\mathrm{H}-\mathrm{F}$ condition, etc.) are not issues. The cost of not incorporating knowledge of $\mathbf{V}$ is that the OLS estimate $\mathbf{b}_{\mathrm{ij}}$ is not necessarily the best estimate of the respective "true" (random) parameter vector $\beta_{\mathrm{ij}}$ for the $\mathrm{jth}$ unit in group. $\mathrm{i}$. This of ten is a negligible cost because ordinarily the between unit variance is larger than within unit variances.

In the case of polynomial regression curves, one could fit successively linear, quadratic, and cubic curves to individual subjects. (Fitting polynomials above degree three rarely produces useful parameter estimates. Even results from fitted third degree polynomials are often of questionable merit.) Analysis of variance can be applied to the subject parameter estimates, typically beginning with the higher order terms, to ascertain the degree of polynomial required. This step is followed by fitting curves of the determined order, and drawing inferences about the treatment groups on the basis of these curves.

Results extracted from using the REPEATED statement in SAS PROC GLM, which provides tests for the significance of parameter estimates from sequentially fitted polynomials, are shown in Table 6. (Tests for higher order effects, which were all nonsignificant, were also printed by the GLM procedure.) These results indicate quadratic models are adequate, with a hint of lack of fit suggested by the average Time Cubic parameter being significantly different from zero at the 0.1163 level. Nonsignificance of the Time Quad*Prog interaction suggests the quadratic models for the three Programs might be forced to have the same quadratic estimate. But this would probably be counterproductive because one would expect the response to the CONT Program to be flat. The Time Linear*Program interaction is significant at the 0.0110 level. This implies that, if straight lines were fitted for the three Programs, they would have significantly different slopes. The significance of the Time Linear effect $(p=0.0028)$ implies the average of the slopes is different from zero. As noted earlier, however, the average trend is probably not of great practical value because the slopes differ between the programs.

Having decided upon the degree of the model, the next step is to obtain equations for fitted models. Unfortunately, the GLM procedure in SAS does not provide parameter estimates directly from this analysis. But parameter estimates for the treatment curves can be obtained by fitting a curve for each subject and then averaging parameter estimates across subjects in the treatment groups. Coefficients for equations so obtained for quadratic curves fitted to the weight training data are shown in Table 7, along with standard deviations of the parameter estimates.

Graphs of the average quadratic curves are plotted in Figure 2, with Program means plotted around them. Predicted values at Times 1-7 from the fitted curves with standard deviations are shown in Table 8 . These predicted values were calculated as averages of predicted values from the individual subject curves. It is usually advantageous to make comparisons between the treatment group at individual Times using these average predicted values rather than using the ordinary means because the predicted values have been smoothed by the fitted curve.

\section{ANALYSIS OF UNBALANCED REPEATED MEASURES DATA}

Unbalanced data occur in repeated measures studies in a variety of ways. Study designs ordinarily call for all subjects to provide measures at the same time points, but complications in the data collection process of ten cause some subjects to not provide data at some points. In other 
studies it might not be feasible to even plan for all subjects to provide data at a common set of time points.

Analysis of variance methods, as generalizations of the split plot in time approach, are discussed by Milliken and Johnson (1984). Applications of these methods usually assume an unbalanced data analog of compound symmetry, because the H-F condition is too complicated to formulate with unbalanced data. With this assumption, analysis of variance of unbalanced repeated measures data falls in the general category of unbalanced mixed linear models. This encompasses a very large and complicated set of problems for data analysis, more than can be discussed here. We therefore refer the reader to Milliken and Johnson. Instead, we focus on procedures specific to repeated measures data.

In order to accommodate unbalanced data, we specify the model

$$
\mathbf{y}_{\mathrm{ij}}=\mathbf{X}_{\mathrm{ij}} \beta_{\mathrm{i}}+\mathbf{e}_{\mathrm{ij}} \text {, }
$$

where $\mathbf{y}_{\mathrm{ij}}$ is the vector of repeated measures and $\mathbf{X}_{\mathrm{ij}}$ is the matrix relating the repeated measures to the time points for the $\mathrm{jth}$ unit in the ith treatment group, $\beta_{\mathrm{i}}$ is the parameter vector for the $i$ th treatment, and $e_{i j}$ is the error vector for the $j$ th unit in the ith group. We must now specify possibly different covariance matrices for each subject, $V\left(e_{i j}\right)=V_{i j}$. This is necessary to reflect the dependence of the covariance matrix on the number and spacing of the repeated measures. Fitting the model to each unit via OLS yields

$$
\mathbf{b}_{\mathrm{ij}}=\left(\mathbf{X}_{\mathrm{ij}} \mathbf{X}_{\mathrm{ij}}\right)^{-1} \mathbf{X}_{\mathrm{ij}} \mathbf{y}_{\mathrm{ij}}
$$

as the vector of parameter estimates for unit $\mathrm{j}$ in group $\mathrm{i}$. Then $\mathrm{b}_{\mathrm{ij}}$ has expectation $\mathrm{E}\left(\mathrm{b}_{\mathrm{ij}}\right)=\beta_{\mathrm{i}}$, and variance $\mathrm{V}\left(\mathrm{b}_{\mathrm{ij}}\right)=\left(\mathbf{X}_{\mathrm{ij}} \mathbf{X}_{\mathrm{ij}}\right)^{-1} \mathbf{X}_{\mathrm{ij}} \mathrm{V}_{\mathrm{ij}} \mathbf{X}_{\mathrm{ij}}\left(\mathbf{X}_{\mathrm{ij}} \mathbf{X}_{\mathrm{ij}}\right)^{-1}$. It follows that the group sample means of the unit estimates are unbiased estimates of the group population mean; i.e. $\mathrm{E}\left(\mathbf{b}_{\mathrm{i}}\right)=\beta_{\mathrm{i}}$, where the ad hoc estimators $\mathbf{b}_{\mathrm{i} .}=\mathrm{n}_{\mathrm{i}}^{-1} \Sigma_{\mathrm{j}}\left(\mathbf{b}_{\mathrm{ij}}\right)$.

The model just described is closely related to the so-called random coefficient regression (RCR) model. Following the description of Vonesh and Carter (1987), $\mathbf{y}_{\mathrm{ij}}=\mathbf{X}_{\mathrm{ij}} \beta_{\mathrm{ij}}+\epsilon_{\mathrm{ij}}$, where $\beta_{\mathrm{ij}}$ is assumed normally distributed with mean $\beta_{\mathrm{i}}$ and covariance $\Gamma$, and $\epsilon_{\mathrm{ij}}$ is normally distributed with mean zero and covariance matrix $\sigma^{2} \mathrm{I}$ of appropriate dimension. This makes $\mathbf{V}_{\mathrm{ij}}=\mathbf{X}_{\mathrm{ij}} \Gamma \mathbf{X}_{\mathrm{ij}}{ }^{\prime}+$ $\sigma^{2} \mathrm{I}$. The RCR model is sometimes criticized for the property that $\mathbf{V}_{\mathrm{ij}}$ depends upon $\mathbf{X}_{\mathrm{ij}}$ in this manner. See Ware (1985). The RCR model is fitted in stages: First, $\mathbf{b}_{\mathrm{ij}}$ is computed as defined above for each subject. Next, an estimator of the covariance matrix of the (combined) vector of regression coefficients for all the subjects is obtained. Then (estimated) generalized least squares is applied to obtain estimates of the $\beta_{\mathrm{i}}$ vectors, along with estimates of their covariances.

Gumpertz and Pantula (1989) proposed using the ad hoc estimators $b_{i}$. They showed that these estimators have near optimal properties even in unbalanced cases. They also showed that the covariance matrix $n^{-1} S_{i}$ is an unbiased estimate of $V\left(b_{i}\right)$, where $S_{i}=\left(n_{i}-1\right)^{-1} \Sigma_{j}\left(b_{i j}-b_{i j}\right)\left(b_{i j}-b_{i}\right)^{\prime}$. Although they stated and proved the covariance result in the context of the random coefficient regression model, it holds for general $V_{\mathrm{ij}}$. This provides a basis for approximate inference using the ad hoc $b_{\mathrm{i}}$. estimators even with unbalanced repeated measures data. Further, the GumpertzPantula covariance result holds for certain other experiment designs.

Considerable effort has been expended in recent years to obtain maximum likelihood estimators for repeated measure models with unbalanced data and structured covariance matrices. See Ware (1985) for a review. Some of these methods have been implemented in the computer program BMDP 5V, employing estimation techniques of Jennrich and Schlucter (1984). The BMDP 5V setup assumes that all units potentially had data at the same points (i.e. the data were potentially balanced) but some subjects have missing data at some points. The primary limiting 
aspect of this formulation is that relatively few different time points are allowed. A variety of covariance structures are permitted, including compound symmetry, 1st order autoregressive, unstructured, and the random coefficient regression model covariance (which is called random effects covariance). In addition to parameter estimates for the fitted models, BMDP 5V also prints estimates to the covariance matrix under the various assumed structures.

Data from the weight training study were used to make a preliminary comparison of estimates using the ad hoc approach with maximum likelihood from BMDP 5V assuming 1st order autoregressive errors. The average regression line $E(y)=\alpha_{0}+\alpha_{1} t+\alpha_{2} t^{2}$ (across programs) was estimated using each of the methods. (The choice to estimate the average regression line was made because BMDP $5 \mathrm{~V}$ estimates this directly, along with estimates of differences of individual program parameters from the average.) This was done for the entire data set, and then data were discarded in various patterns: $20 \%, 40 \%$ and $60 \%$ were discarded (in sequence) at random in the Subject by Time array. Then $20 \%, 40 \%$ and $60 \%$ were discarded randomly across subjects, but with the condition that all missing points are at the end of the series. Thus some subjects have complete data, some are missing the last time, some are missing the last two times, etc. Estimates and standard errors appear in Table 9.

Results in this Table 9 indicate that the ad hoc method performs well relative to ML unless a large fraction of data is missing, on the order of $50 \%$ or more. Performance of the ad hoc method is especially poor when a large amount of data is missing at the last end of the series.

\section{REFERENCES}

Box, G.E.P. (1954), "Some Theorems on Quadratic Forms Applied in the Study of Analysis of Variance Problems," Annals of Mathematical Statistics, 25, 290-302.

COLE, J.W.L. AND GRIzzLE, J.E. (1966), "Applications of Multivariate Analysis of Variance to Repeated Measurements Experiments," Biometrics, 22, 810-828.

Freund, R.J., LitTell, R.C. ANd SPECtor, P.C. (1986), SAS System for Linear Models, Cary, NC, SAS Institute Inc.

Greenhouse, S.W. And Geisser, S. (1959), "On Methods in the Analysis of Profile Data," Psychometrika, 24, 95-112.

Grizzle, J.E. And Allen, D.M. (1969), "Analysis of Growth and Dose Response Curves," Biometrics, 25, 357-381.

Gumpertz, M. And Pantula, S.G. (1989), "A Simple Approach to Inference in Random Coefficient Models," The American Statistician, 42, 203-210.

HUYNh, H. AND FELDT, L.S. (1970), "Conditions under Which Mean Square Ratios in Repeated Measurements Designs Have Exact F-Distributions," Journal of the American Statistical Association, 65, 1582-1589.

JennRICh, R.I. AND Schlucter, M.D. (1986), "Unbalanced Repeated Measures Models with Structured Covariance Matrices," Biometrics, 42, 805-820.

LoONEy, S.W. AND StANLEy, W.B. (1989), "Exploratory Repeated Measures Analysis for Two or More Groups," The American Statistician, 43, 220-225.

Milliken, G.A. (1990), "Analysis of Repeated-Measures Designs," in Statistical Methodology in the Pharmaceutical Sciences, D.A. Berry, ed., New York: Marcel Dekker, Inc., 83-116.

Milliken, G.A. And Johnson, D.E. (1984), Analysis of Messy Data, New York: Van Nostrand Reinhold Company.

WARE, J.H. (1985), "Linear Models for the Analysis of Longitudinal Studies," The American Statistician, 39, 95-101. 
Table 1. Data for Exercise Therapy Study

\begin{tabular}{|c|c|c|c|c|c|c|c|c|}
\hline OBS & PROGRAM & $\underline{\mathrm{S}} 1$ & $\underline{\mathrm{S}}$ & $\underline{\mathrm{S} 3}$ & $\underline{\mathrm{S} 4}$ & $\underline{\mathrm{S}}$ & $\underline{\$ 6}$ & $\underline{57}$ \\
\hline 1 & CONT & 85 & 85 & 86 & $\overline{85}$ & $\overline{87}$ & $\overline{86}$ & $\overline{87}$ \\
\hline 2 & CONT & 80 & 79 & 79 & 78 & 78 & 79 & 78 \\
\hline 3 & CONT & 78 & 77 & 77 & 77 & 76 & 76 & 77 \\
\hline 4 & CONT & 84 & 84 & 85 & 84 & 83 & 84 & 85 \\
\hline 5 & CONT & 80 & 81 & 80 & 80 & 79 & 79 & 80 \\
\hline 6 & CONT & 76 & 78 & 77 & 78 & 78 & 77 & 74 \\
\hline 7 & CONT & 79 & 79 & 80 & 79 & 80 & 79 & 81 \\
\hline 8 & CONT & 76 & 76 & 76 & 75 & 75 & 74 & \\
\hline 9 & CONT & 77 & 78 & 78 & 80 & 80 & 81 & 80 \\
\hline 10 & CONT & 79 & 79 & 79 & 79 & 77 & 78 & \\
\hline 11 & CONT & 81 & 81 & 80 & 80 & 80 & 81 & \\
\hline 12 & CONT & 77 & 76 & 77 & 78 & 77 & 77 & 77 \\
\hline 13 & CONT & 82 & 83 & 83 & 83 & 84 & 83 & 83 \\
\hline 14 & CONT & 84 & 84 & 83 & 82 & 81 & 79 & 78 \\
\hline 15 & CONT & 79 & 81 & 81 & 82 & 82 & 82 & 80 \\
\hline 16 & CONT & 79 & 79 & 78 & 77 & 77 & 78 & 78 \\
\hline 17 & CONT & 83 & 82 & 83 & 85 & 84 & 83 & 82 \\
\hline 18 & CONT & 78 & 78 & 79 & 79 & 78 & 77 & 77 \\
\hline 19 & CONT & 80 & 80 & 79 & 79 & 80 & 80 & 80 \\
\hline 20 & CONT & 78 & 79 & 80 & 81 & 80 & 79 & 80 \\
\hline 1 & RI & 79 & 79 & 79 & 80 & 80 & 78 & 80 \\
\hline 2 & RI & 83 & 83 & 85 & 85 & 86 & 87 & 87 \\
\hline 3 & RI & 81 & 83 & 82 & 82 & 83 & 83 & 82 \\
\hline 4 & RI & 81 & 81 & 81 & 82 & 82 & 83 & 81 \\
\hline 5 & RI & 80 & 81 & 82 & 82 & 82 & 84 & 86 \\
\hline 6 & $\mathrm{RI}$ & 76 & 76 & 76 & 76 & 76 & 76 & 75 \\
\hline 7 & $\mathrm{RI}$ & 81 & 84 & 83 & 83 & 85 & 85 & 85 \\
\hline 8 & RI & 77 & 78 & 79 & 79 & 81 & 82 & 81 \\
\hline 9 & RI & 84 & 85 & 87 & 89 & 88 & 85 & 86 \\
\hline 10 & $\mathrm{RI}$ & 74 & 75 & 78 & 78 & 79 & 78 & 78 \\
\hline 11 & RI & 76 & 77 & 77 & 77 & 77 & 76 & 76 \\
\hline 12 & RI & 84 & 84 & 86 & 85 & 86 & 86 & 86 \\
\hline 13 & RI & 79 & 80 & 79 & 80 & 80 & 82 & 82 \\
\hline 14 & RI & 78 & 78 & 77 & 76 & 75 & 75 & 76 \\
\hline 15 & RI & 78 & 80 & 77 & 77 & 75 & 75 & 75 \\
\hline 16 & $\mathrm{RI}$ & 84 & 85 & 85 & 85 & 85 & 83 & 82 \\
\hline 1 & WI & 84 & 85 & 84 & 83 & 83 & 83 & 84 \\
\hline 2 & WI & 74 & 75 & 75 & 76 & 75 & 76 & 76 \\
\hline 3 & WI & 83 & 84 & 82 & 81 & 83 & 83 & 82 \\
\hline 4 & WI & 86 & 87 & 87 & 87 & 87 & 87 & 86 \\
\hline 5 & WI & 82 & 83 & 84 & 85 & 84 & 85 & 86 \\
\hline 6 & WI & 79 & 80 & 79 & 79 & 80 & 79 & 80 \\
\hline 7 & WI & 79 & 79 & 79 & 81 & 81 & 83 & 83 \\
\hline 8 & WI & 87 & 89 & 91 & 90 & 91 & 92 & 92 \\
\hline 9 & WI & 81 & 81 & 81 & 82 & 82 & 83 & 83 \\
\hline 10 & WI & 82 & 82 & 82 & 84 & 86 & 85 & 87 \\
\hline 11 & WI & 79 & 79 & 80 & 81 & 81 & 81 & 81 \\
\hline 12 & WI & 79 & 80 & 81 & 82 & 83 & 82 & 82 \\
\hline 13 & WI & 83 & 84 & 84 & 84 & 84 & 83 & 83 \\
\hline
\end{tabular}


Table 2. Means and Standard Deviations for Exercise Therapy Data

\begin{tabular}{|c|c|c|c|c|c|c|c|c|}
\hline \multirow{2}{*}{$\frac{\text { PROGRAM }}{\text { CONT }}$} & & STR1 & STR2 & $\underline{\operatorname{STR} 3}$ & STR4 & STR5 & STR6 & STR7 \\
\hline & mean & 79.8 & 80.0 & 80.0 & 80.1 & 79.8 & 79.6 & 79.6 \\
\hline & std dev & 2.67 & 2.63 & 2.75 & 2.74 & 3.04 & 2.95 & 3.25 \\
\hline RI & mean & 79.7 & 80.6 & 80.8 & 81.0 & 81.25 & 81.13 & 81.13 \\
\hline & std dev & 3.11 & 3.22 & 3.58 & 3.79 & 4.12 & 4.13 & 4.18 \\
\hline WI & mean & 81.0 & 81.7 & 81.9 & 82.5 & 82.6 & 82.7 & 83.1 \\
\hline & std dev & 3.11 & 3.35 & 3.48 & 3.04 & 3.34 & 3.27 & 3.35 \\
\hline
\end{tabular}

Table 3. Univariate Analysis of Variance for Weight Training Data

\begin{tabular}{|c|c|c|c|c|}
\hline \multirow[b]{2}{*}{ Source of Variation } & \multirow[b]{2}{*}{$\underline{\mathrm{df}}$} & \multirow[b]{2}{*}{ Mean Square } & \multicolumn{2}{|c|}{ Univariate } \\
\hline & & & $\underline{F}$ & $\underline{p}$ \\
\hline Program & 2 & 209.72 & 3.07 & 0.0548 \\
\hline $\begin{array}{l}\text { Error(a) }= \\
\text { Subj.(Program) }\end{array}$ & 54 & 86.42 & & \\
\hline $\begin{array}{l}\text { Time } \\
\text { Program*Time }\end{array}$ & $\begin{array}{r}6 \\
12\end{array}$ & $\begin{array}{l}8.89 \\
3.58\end{array}$ & $\begin{array}{l}7.43 \\
2.99\end{array}$ & $\begin{array}{l}0.0001^{*} \\
0.0005^{*}\end{array}$ \\
\hline $\begin{array}{l}\text { Error(b) }= \\
\text { Subj"Time(Prog) }\end{array}$ & 324 & 1.20 & & \\
\hline
\end{tabular}

Table 4. Multivariate Tests (Pillai's Trace) for Weight Training Data

\begin{tabular}{lcc} 
Effect & Multivariate F & \multicolumn{2}{c}{ Significance Probability P } \\
Time & 6.46 & 0.0001 \\
Time*Program & 1.37 & 0.1943
\end{tabular}

Table 5. Summary of Univariate, Multivariate and Adjusted Univariate P-values

\begin{tabular}{|c|c|c|c|}
\hline$\underline{\text { Effect }}$ & Univariate & Multivariate & Adjusted Univariate \\
\hline Time & 0.0001 & 0.0001 & 0.0001 \\
\hline Time*Program & 0.0005 & 0.1943 & 0.0130 \\
\hline
\end{tabular}


Table 6. Analysis of Variance of Polynomial Trends

\begin{tabular}{lrrrc} 
Source of Variation & df & Mean Square & F & D \\
\cline { 5 - 6 } Time Linear & 1 & 40.514 & 9.85 & 0.0028 \\
Time Lin*Prog & 2 & 20.196 & 4.91 & 0.0110 \\
Error & 54 & 4.115 & & \\
Time Quadratic & 1 & 10.577 & 8.64 & 0.0048 \\
Time Quad*Prog & 2 & 0.712 & 0.58 & 0.5626 \\
Error & 54 & 1.225 & & \\
Time Cubic & 1 & 1.313 & 2.55 & 0.1163 \\
Time Cub*Prog & 2 & 0.020 & 0.04 & 0.9620 \\
Error & 54 & 0.516 & &
\end{tabular}

Table 7. Average Parameter Estimates and Standard Deviations

\begin{tabular}{c|ccc} 
PROGRAM & INTERCEPT & LINEAR & QUADRATIC \\
\hline CONT & $79.61(3.13)$ & $0.223(1.153)$ & $-0.034(0.137)$ \\
RI & $79.08(3.14)$ & $0.793(1.192)$ & $-0.073(0.134)$ \\
WI & $80.52(3.39)$ & $0.601(0.813)$ & $-0.035(0.089)$
\end{tabular}

Table 8. Estimates and Contrasts of Quadratic Curves

\begin{tabular}{ccccccccc} 
PROGRAM & & STR1 & STR2 & STR3 & STR4 & STR5 & STR6 & STR7 \\
CONT & mean & 79.8 & 79.9 & 80.0 & 80.0 & 79.9 & 79.7 & 79.5 \\
\multirow{2}{*}{ RI } & std dev & 2.70 & 2.60 & 2.67 & 2.77 & 2.85 & 2.96 & 3.21 \\
& mean & 79.8 & 80.4 & 80.8 & 81.1 & 81.2 & 81.2 & 81.1 \\
WI & std dev & 3.08 & 3.27 & 3.53 & 3.56 & 3.92 & 4.05 & 4.23 \\
& mean & 81.1 & 81.6 & 82.0 & 82.4 & 82.7 & 82.9 & 83.1 \\
CONTRAST & std dev & 3.22 & 3.19 & 3.21 & 3.24 & 3.25 & 3.26 & 3.31 \\
CONT VS TRT & & & & & & & & \\
RI VS WI & P-value & 0.44 & 0.21 & 0.11 & 0.06 & 0.03 & 0.02 & 0.01 \\
& P-value & 0.20 & 0.23 & 0.25 & 0.24 & 0.20 & 0.15 & 0.10
\end{tabular}


Table 9. Comparison of Maximum Likelihood and ad hoc Parameter Estimates

$\begin{array}{ccccc}\text { Missing data pattern } & \text { Estimation Method } & \alpha_{0} & \alpha_{1} & \alpha_{2} \\ \text { Complete } & \text { ML } & 79.66(0.46) & 0.55(0.14) & -0.05(0.02) \\ \text { ad hoc } & 79.74(0.43) & 0.54(0.14) & -0.05(0.02) \\ \text { random 20\% } & \text { ML } & 79.59(0.48) & 0.63(0.16) & -0.06(0.02) \\ & \text { ad hoc } & 79.68(0.45) & 0.65(0.19) & -0.07(0.02) \\ \text { random 40\% } & \text { ML } & 79.67(0.05) & 0.66(0.17) & -0.06(0.02) \\ \text { ad hoc } & \text { ML } & 80.11(0.65) & 0.54(0.23) & -0.05(0.03) \\ \text { random 60\% } & \text { ad hoc } & 79.69(0.55) & 0.59(0.21) & -0.05(0.03) \\ & & 79.78(0.83) & 0.73(0.33) & -0.07(0.02) \\ \text { last 20\% } & & & \\ \text { ML } & \text { ad hoc } & 79.61(0.46) & 0.61(0.15) & -0.06(0.02) \\ \text { last 40\% } & \text { ML } & 79.61(0.42) & 0.65(0.17) & -0.07(0.02) \\ & \text { ad hoc } & 79.58(0.47) & 0.70(0.19) & -0.08(0.03) \\ \text { last 60\% } & \text { ML } & 79.63(0.44) & 0.74(0.20) & -0.09(0.04) \\ & \text { ad hoc } & 79.54(0.49) & 0.74(0.28) & -0.08(0.06) \\ & & 79.22(0.67) & 1.34(0.44) & -0.22(0.12)\end{array}$


Figure 1. Means for Exercise Therapy Programs

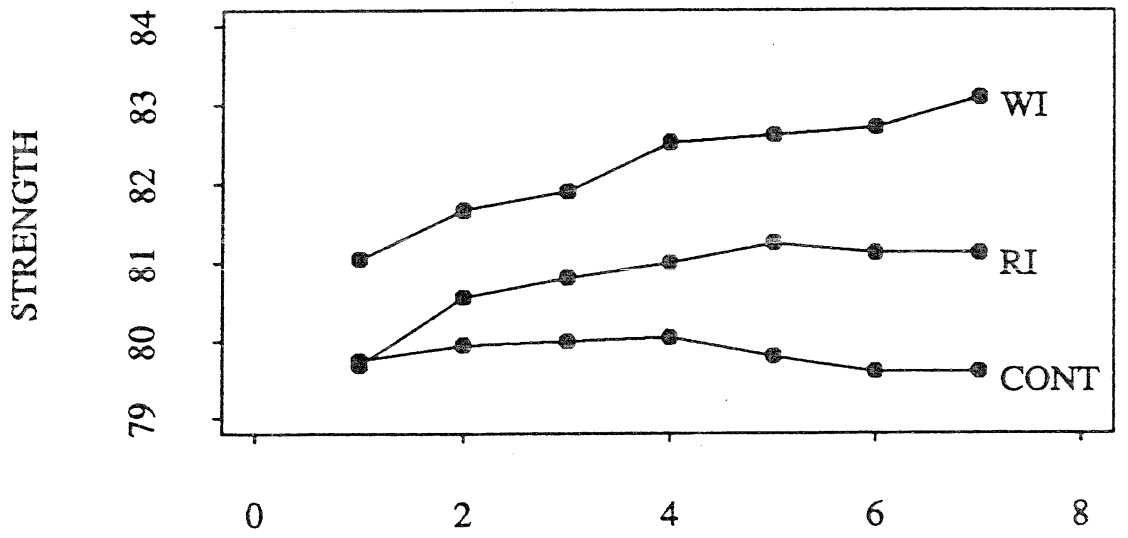

TIME

Figure 2. Quadratic Regression Curves for Exercise Therapy Programs

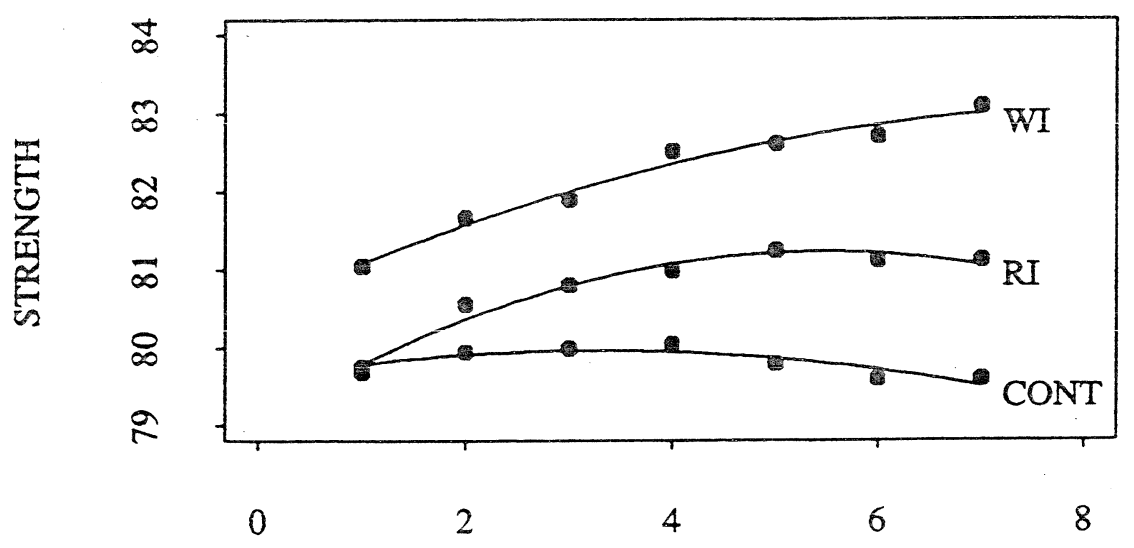

TIME 